Article

\title{
Enhancing Dark Shade Pigment Dyeing of Cotton Fabric Using Plasma Treatment
}

\author{
Chi-Wai Kan * and Wai-Shan Man \\ Institute of Textiles and Clothing, The Hong Kong Polytechnic University, Hung Hom, Kowloon, \\ Hong Kong 999077, China; wsman@yahoo.com \\ * Correspondence: tccwk@polyu.edu.hk; Tel.: +852-2766-6531; Fax: +852-2773-1432
}

Received: 19 June 2017; Accepted: 16 July 2017; Published: 19 July 2017

\begin{abstract}
This study is intended to investigate the effect of atmospheric pressure plasma treatment on dark shade pigment dyeing of cotton fabric. Experimental results reveal that plasma-treated cotton fabric can attain better color yield, levelness, and crocking fastness in dark shade pigment dyeing, compared with normal cotton fabric (not plasma treated). SEM analysis indicates that cracks and grooves were formed on the cotton fiber surface where the pigment and the binder can get deposited and improve the color yield, levelness, and crocking fastness. It was also noticed that pigment was aggregated when deposited on the fiber surface which could affect the final color properties.
\end{abstract}

Keywords: atmospheric plasma treatment; dark shade; pigment dyeing; cotton fabric

\section{Introduction}

Pigments offer superior advantages over dyes, such as shorter dyeing time, low chemicals usage, less effluent, and being non-fiber selective [1]. Generally speaking, pigments provide good light and washing fastness but they always have comparatively poor color yield, levelness, and crocking fastness. In previous studies [2,3], plasma treatment has been found to be an effective way for improving color yield, levelness, and crocking fastness of pigment dyed fabrics. During plasma treatment, the fiber surface can be modified physically [4-6] and chemically [7-9]. In physical modification, active plasma species bombarding the fiber surface causes ejection of atoms on the fiber surface [10-12]. Cracks and grooves are formed on the fiber surface which helps improve the wicking and absorption of water in the plasma treated fiber [2,3]. On the other hand, when oxygen is used as the plasma gas, it can help introduce oxygen-containing functional groups such as $-\mathrm{OH}$ and $-\mathrm{C}=\mathrm{O}$, which are hydrophilic in nature, into the fiber surface $[2,11,12]$. Plasma-induced physical and chemical actions help to improve the pigment dyeing behavior of cotton fabric [2,3]. In industrial applications of pigment dyeing on cotton with dark shade, the dyeing results may not be as good for light and medium shades. In our previous study [3], the highest pigment concentration used was $5 \mathrm{~g} / \mathrm{L}$ but in this study, the pigment concentration is $40 \mathrm{~g} / \mathrm{L}$. Therefore, the effect of oxygen plasma treatment on dark shade pigment dyeing of cotton fabric has been studied. Effects on properties such as color yield, levelness, crocking fastness, and dry-cleaning fastness are reported.

\section{Experimental}

\subsection{Material}

Ready-for-dyeing plain weave cotton fabric of $250 \mathrm{~g} / \mathrm{m}^{2}$ with $0.52 \mathrm{~mm}$ thickness was used to investigate the effect of plasma on dark shade pigment dyeing. Fabric was washed with dilute acetone (99\%, GR Grade) for $5 \mathrm{~min}$ and subsequently dried in an oven at $50{ }^{\circ} \mathrm{C}$ for $10 \mathrm{~min}$. Samples were conditioned under $65 \% \pm 2 \%$ relative humidity and $20 \pm 2{ }^{\circ} \mathrm{C}$ for at least $24 \mathrm{~h}$ prior to use. 


\subsection{Atmospheric Pressure Plasma Treatment}

Cotton fabric was treated with atmospheric pressure plasma using a plasma jet (APPJ) with a rectangular nozzle (Atomflo 400, AH-500L, Surfx Technologies LLC, Redondo Beach, CA, USA) which covered an active area of $50 \times 20 \mathrm{~mm}^{2}$. The plasma nozzle was mounted above the cotton fabric and plasma reactive species were exposed to the fabric vertically. Plasma was generated at $13.56 \mathrm{MHz}$ radio frequency. The setup for plasma treatment is shown in Figure 1. Helium (He, 99.995\% purity) and oxygen $\left(\mathrm{O}_{2}, 99.7 \%\right.$ purity) were applied as carrier and reactive gases respectively in the plasma treatment. The helium flow rate was fixed at $30 \mathrm{~L} / \mathrm{min}$. Values of discharge power, oxygen flow rate, and jet moving speed are listed in Table 1 . After plasma treatment, treated fabric was conditioned at a relative humidity of $65 \pm 2 \%$ at $20 \pm 2{ }^{\circ} \mathrm{C}$ for $24 \mathrm{~h}$ before use.

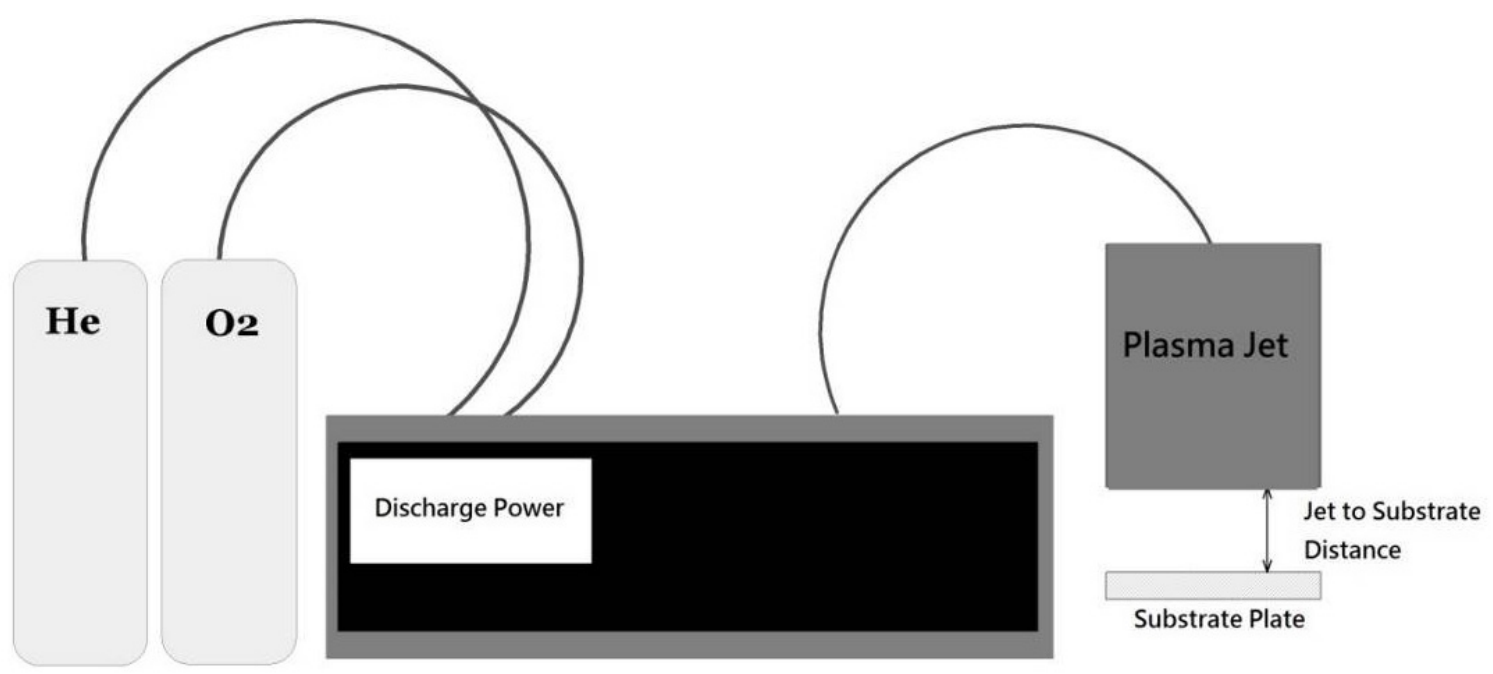

Figure 1. Schematic diagram for atmospheric pressure plasma treatment.

Table 1. Operational parameters settings for plasma treatment.

\begin{tabular}{ccccc}
\hline $\begin{array}{c}\text { Sample } \\
\text { Code }\end{array}$ & $\begin{array}{c}\text { Discharge Power } \\
(\mathbf{W})\end{array}$ & $\begin{array}{c}\text { Oxygen Flow Rate } \\
(\mathrm{L} / \mathbf{m i n})\end{array}$ & $\begin{array}{c}\text { Jet Moving Speed } \\
(\mathbf{m m} / \mathbf{s})\end{array}$ & $\begin{array}{c}\text { Jet to Substrate } \\
\text { Distance }(\mathbf{m m})\end{array}$ \\
\hline 1 & 130 & 0.3 & 5 & 3 \\
2 & 150 & 0.3 & 5 & 3 \\
3 & 170 & 0.3 & 5 & 3 \\
4 & 170 & 0.2 & 5 & 3 \\
5 & 170 & 0.4 & 5 & 3 \\
6 & 170 & 0.6 & 5 & 3 \\
7 & 150 & 0.4 & 1 & 3 \\
8 & 150 & 0.4 & 5 & 3 \\
9 & 150 & 0.4 & 9 & \\
\hline
\end{tabular}

\subsection{Pigment Dyeing Solution}

Blue pigment dyeing solution $(40 \mathrm{~g} / \mathrm{L})$ for dark shade was prepared (as recommended by the supplier) by mixing $40 \mathrm{~g}$ Printofix Pigment (supplied by Archroma, Shanghai, China) and $170 \mathrm{~g}$ Printofix Binder (supplied by Archroma) in $1 \mathrm{~L}$ of deionized water. The pigment dyeing solution was well stirred in an ultrasound machine until no solid pigment particle was noted. 


\subsection{Particle Size Analysis}

After preparing the pigment dyeing suspension, the effective diameter of the blue pigment particle was measured using a Particle Size Analyzer (Zeta Potential Analyzer, Brookhaven Instruments Corporation, Holtsville, NY, USA).

\subsection{Pigment Dyeing}

The pigment dyeing of cotton fabrics was conducted using a pad-dry cure process. The fabrics were padded with pigment with a horizontal padding machine (Weriner Mathis AG, Oberhasli, Switzerland) for an uptake of $80 \%$. The padded fabrics were dried at $90{ }^{\circ} \mathrm{C}$ for $3 \mathrm{~min}$ and then cured at $145{ }^{\circ} \mathrm{C}$ for $5 \mathrm{~min}$ using a curing machine. After dyeing, the fabrics were conditioned at relative humidity of $65 \pm 2 \%$ and a temperature of $20 \pm 2{ }^{\circ} \mathrm{C}$ for $24 \mathrm{~h}$ prior to evaluation.

\subsection{Scanning Electron Microscopy (SEM)}

The surface features of cotton fabrics were observed using scanning electron microscope (SEM, JSM-6490, JEOL Ltd., Tokyo, Japan); 4000× magnification images of substrates were captured with accelerating voltage of $20 \mathrm{kV}$.

\subsection{Color Yield Measurement}

Color yield of the pigment dyed cotton fabric was characterized by reflectance measurement at given wavelength intervals $(400-700 \mathrm{~nm}$ ) throughout the visible spectrum using a spectrophotometer (Macbeth Color-Eye 7000A, Singapore). The fabric reflectance was measured under illuminant D65 and $10^{\circ}$ standard observer techniques. The fabric was folded four times to ensure opacity and reflectance measurements from 400 to $700 \mathrm{~nm}$ at $20 \mathrm{~nm}$ intervals were obtained. The reflectance values were then converted into $K / S$ values according to the Kubelka-Munk equation (Equation (1)).

$$
\frac{K}{S}=\frac{(1-R)^{2}}{2 R}
$$

where $K$ is the absorption coefficient of the colorant, $S$ is the scattering coefficient of the colored substrate and $R$ is the reflectance of the colored sample. The higher the $K / S$ value, the greater is the pigment uptake, resulting in better color yield.

For comparing color yields of different samples, a $K / S_{\text {Sum }}$ value was calculated by summation of individual $K / S$ values over the visible spectrum wavelength $(400-700 \mathrm{~nm})$. The higher the $K / S_{\text {Sum }}$ value, the better is the color yield. Based on the $K / S_{\text {Sum }}$ value, the percentage change in color yield under the influence of plasma treatment can be calculated using Equation (2):

$$
\text { Percentage change in color yield }(\%)=\frac{\left(K / S_{\text {Sum }}\right)_{f}}{\left(K / S_{\text {Sum }}\right)_{i}} \times 100 \%
$$

where $\left(K / S_{\text {Sum }}\right)_{i}$ and $\left(K / S_{\text {Sum }}\right)_{f}$ are the color yield of pigment dyed fabric without and with plasma treatment of the fabric, respectively.

\subsection{Levelness}

The levelness of pigment dyed fabric was evaluated using a spectrophotometric method in quantitative terms by establishing a relative unlevelness index (RUI) [2,3]. Reflectance values at 8 different points on the sample swatch were measured using spectrophotometry (Macbeth Color-Eye $7000 \mathrm{~A})$ with D65 illuminant and $10^{\circ}$ standard observer within the visible spectrum $(400-700 \mathrm{~nm})$. The RUIs for the pigment dyed fabric samples were calculated using Equations (3) and $(4)[2,3,13]$. 


$$
S_{\lambda}=\sqrt{\frac{\sum_{i=1}^{n}\left(R_{i}-R_{m}\right)^{2}}{n-1}}
$$

where $S_{\lambda}$ is standard deviation of reflectance values, $R_{i}$ is reflectance value of the $i$ th measurement for each wavelength and $R_{m}$ is mean of reflectance values of $n$ measurements for each wavelength [13].

$$
\mathrm{RUI}=\sum_{400}^{700} C_{\lambda} V_{\lambda}=\sum_{400}^{700} \frac{S_{\lambda}}{R} V_{\lambda}
$$

where $V_{\lambda}$ is photopic relative luminous efficiency function.

The degree of levelness is described according to the RUI value obtained; the suggested interpretations of RUI values are shown in Table 2 [13].

Table 2. Suggested interpretations of RUI values [13].

\begin{tabular}{cc}
\hline$R \boldsymbol{R}$ I & Visual Appearance of Levelness \\
\hline$<0.2$ & Excellent levelness \\
$0.2-0.49$ & Good levelness \\
$0.5-1.0$ & Poor levelness \\
$>1.0$ & Bad levelness \\
\hline
\end{tabular}

\subsection{Color Fastness Evaluation}

Color fastness to crocking and dry-cleaning were measured by AATCC Test Method 8-2016 (color fastness to crocking: crockmeter method) [14] and AATCC Test Method 132-2013 (color fastness to dry-cleaning) [15], respectively.

\section{Results and Discussion}

\subsection{Color Yield and Levelness}

Color yield results of blue pigment dyed cotton fabrics are presented in $K / S_{\text {Sum }}$ which is measured by the spectrophotometer (Table 3).

Table 3. $K / S_{\text {Sum }}$ values and levelness of pigment dyed cotton fabric (the results were measured with

\begin{tabular}{|c|c|c|c|c|c|c|c|c|c|c|}
\hline Sample & $\begin{array}{c}\text { Plasma } \\
\text { Untreated }\end{array}$ & 1 & 2 & 3 & 4 & 5 & 6 & 7 & 8 & 9 \\
\hline$K / S_{\text {Sum }}{ }^{*}$ & $100 \%$ & $113.3 \%$ & $114.2 \%$ & $115.4 \%$ & $112.6 \%$ & $113.4 \%$ & $111.5 \%$ & $111.1 \%$ & $111.3 \%$ & $110.9 \%$ \\
\hline Levelness & 0.42 & 0.25 & 0.21 & 0.21 & 0.24 & 0.25 & 0.26 & 0.26 & 0.28 & 0.29 \\
\hline
\end{tabular}
$5 \%$ tolerance).

* Percentage change (\%) is used for expressing the increase of color yield, $100 \%$ is marked as the $K / S_{\text {Sum }}$ value of pigment dyed untreated fabric (i.e., without plasma treatment). If the percentage is greater than $100 \%$, it means increased color yield.

Table 3 indicates that $K / S_{\text {Sum }}$ values increase with the increase of discharge power in pigment dyeing. When the discharge power is increases from 130 to $170 \mathrm{~W}$, formation of highly active species results in increased etching and oxidation rate [11,12]. Meanwhile, with the use of higher discharge power will allow active species to have higher energy to bombard and interact the substrate and generate more cracks. High level of discharge results in deep cracks and grooves on fibers [2,3]. The formation of cracks and grooves may help to improve the wicking property of the cotton fabric $[2,3]$. In addition, the formation of cracks and grooves increases the surface area also. Thus the distinctive difference in fiber morphology explains that pigment attachment is improved by larger surface area as well as the improved wicking ability. 
The color yield increased with increase of oxygen flow rate and reached the highest at $0.4 \mathrm{~L} / \mathrm{min}$. Unlike the previous study on blue pigment [2], too high a rate of oxygen flow cannot achieve better color yield. With a low oxygen flow rate, the plasma will contain fewer amount of oxygen species which is not sufficient to modify surface hydrophilicity and also the oxidation due to air impurities such as nitrogen and water prevails over the surface modification with oxygen because plasma treatment was conducted at atmospheric pressure, which is not an air-free environment [2]. Meanwhile, with the high oxygen flow rate, it does not much improve the color yield because the collision between oxygen plasma species may occur and this reduces the probability of active oxygen plasma species reacting with the cotton fiber surface [16]. However, with the use of oxygen in this study, the oxidation of cotton fiber surface caused by reactive oxygen plasma species greatly enhances the adhesion forces between cotton fiber surface and water molecules by inducing new functional groups such as $-\mathrm{OH}$, $-\mathrm{C}=\mathrm{O}$, and $-\mathrm{COOH}[2,3,7-9]$. The improvement of water molecule absorption makes the dyeing process more effective.

When the jet moving speed is taken into consideration, active plasma species are allowed to accumulate on the fiber surface for different physical and chemical reactions at low jet moving speed. However, at a high jet moving speed, the active plasma species may not have sufficient time to react with the fiber surface. If a low jet moving speed is used, although the active plasma species may have more time to interact with the fiber surface, the concentration of active species from the plasma jet accumulating on the fiber surface may be increased. Once the concentration of the active species reaches a critical level, further supply of active species may increase the interaction between the active species and the fiber surface but the collision between the active species lowers the energy of active species. Thus, the surface modification effect may become stable, with no further improvement $[5,17]$. So, the jet moving speed of $5 \mathrm{~mm} / \mathrm{s}$ provides the best color yield.

As shown in Table 3, the atmospheric pressure plasma treatment can not only improve the color yield but also improve the levelness of pigment dyed cotton fabrics. For the untreated cotton fabric, the levelness is above 0.4 but it drops to below 0.3 after plasma treatment. The poor levelness of the colored product in pigment dyed fabric is a practical problem which may be due to the pigment molecules agglomeration and migration. Water insoluble pigment molecules can aggregate easily in aqueous solution. After plasma treatment, formation of cracks in the fiber surface and improved surface hydrophilicity help trap pigment in the fiber, leading to better levelness [2,3].

\subsection{Surface Morphological Analysis}

Although color yield and levelness of pigment dyeing improves after plasma treatment, the improvement varies with process parameters of the treatment. Figure 2 shows the surface of pigmented dyed fibers, which is covered by a binder layer. Fiber surface is generally smooth after pigment dyeing. Granular pigments are under the binder layer and the pigment density varies according to plasma conditions. Some pigment particles get stuck on cotton fibers in dark shade pigment dyeing. The number of observable pigment particles gradually increases with the increase in discharge power as shown in Figure $2 b(130 \mathrm{~W})$, Figure $2 \mathrm{c}(150 \mathrm{~W})$, and Figure $2 \mathrm{~d}(170 \mathrm{~W})$, which explains the result in color yield measurement (Table 3), i.e., high $K / S_{\text {Sum }}$ value is given when discharge power level is increasing.

Figure $2 \mathrm{~h}$ shows that pigments are attached on $1 \mathrm{~mm} / \mathrm{s}$ jet moving speed plasma treated fiber, the $\mathrm{K} / S_{\text {Sum }}$ value of $1 \mathrm{~mm} / \mathrm{s}$ plasma treated fabric is relatively low, and the RUI value is high. This SEM image suggests that uneven pigment dispersion and migration during drying happen spontaneously.

There is nearly no morphological difference between the control fiber (Figure 2a) and plasma treated fiber: $9 \mathrm{~mm} / \mathrm{s}$ jet speed (Figure 2j). Effective plasma modification cannot be achieved if the treatment duration is too short. As a result, the improvements on color yield are not significant. 


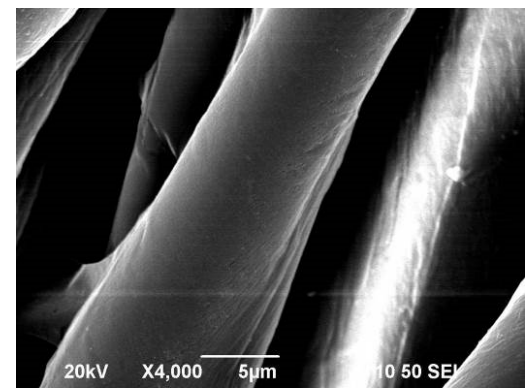

(a)

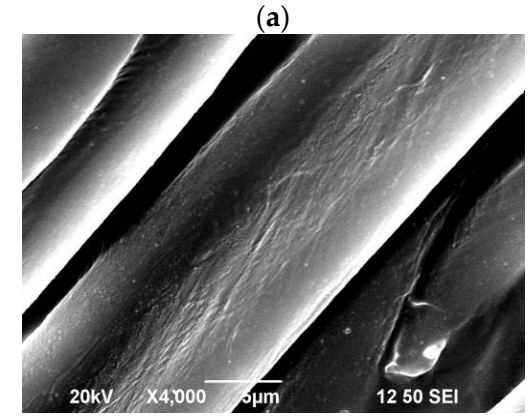

(c)

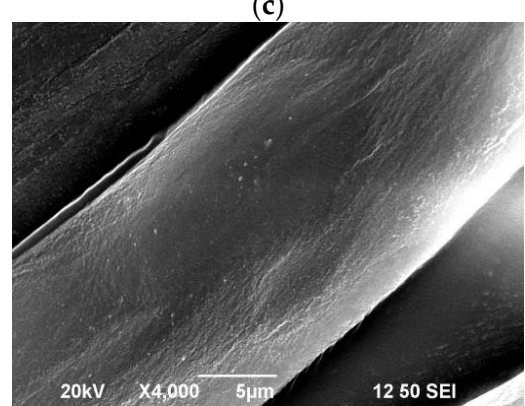

(e)

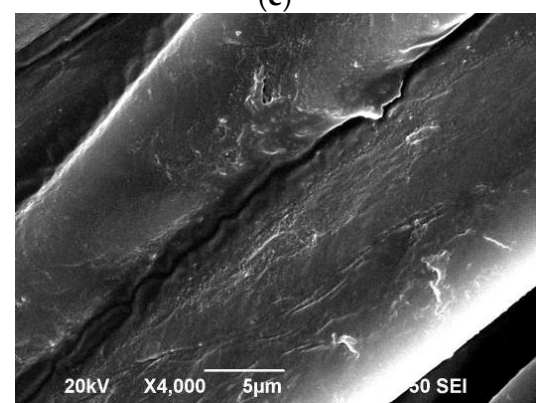

(g)

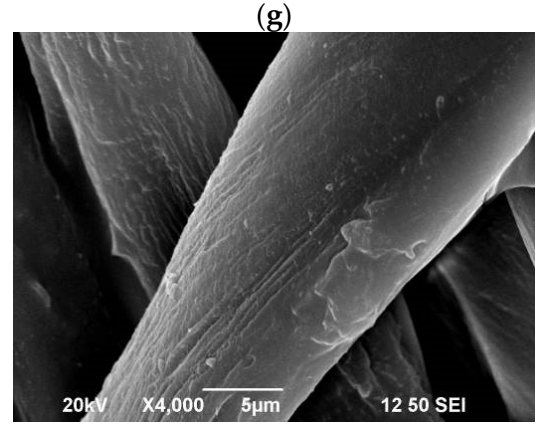

(i)

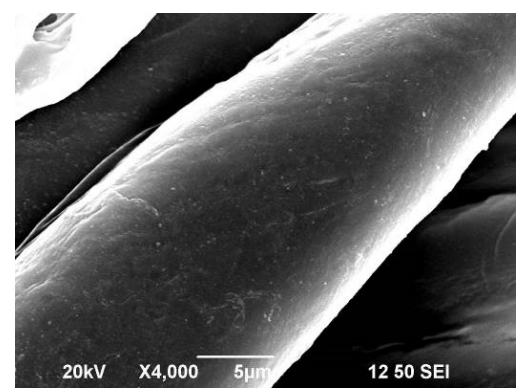

(b)

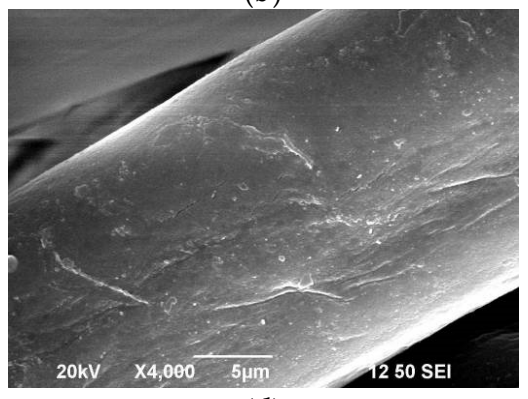

(d)

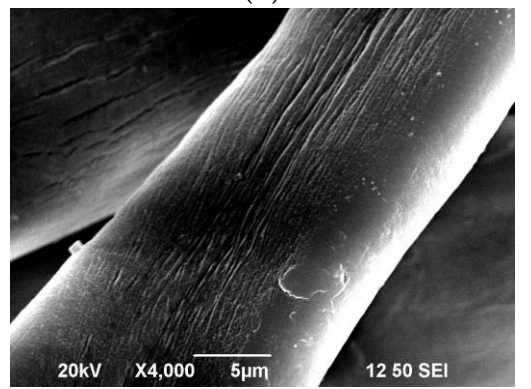

(f)

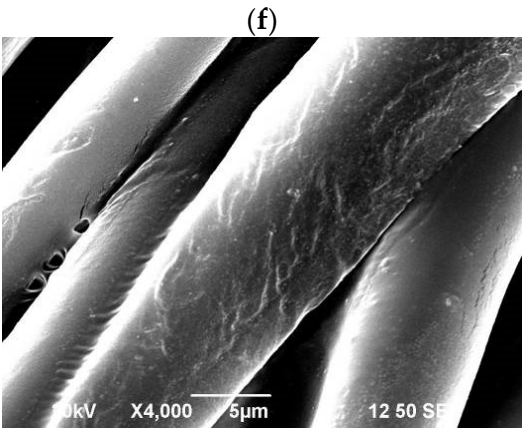

(h)

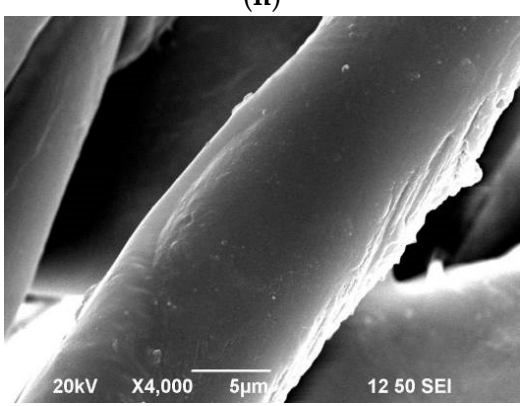

(j)

Figure 2. SEM images of (a) untreated cotton fiber; plasma treated cotton fiber with (b) sample 1; (c) sample 2; (d) sample 3; (e) sample 4; (f) sample 5; (g) sample 6; (h) sample 7; (i) sample 8; and (j) sample 9 with respective treatment parameter settings after pigment dyeing. 
Meanwhile, pigment agglomeration and migration in dark shade pigment dyeing may occur due to high pigment concentration. As illustrated in Figure 3, different sizes of pigment particles are laying on the fiber and most of them are larger than the original size (184.6 nm effective diameter). The calculated diameters of circle and square indicate that pigments are of size 277.8 and $185.4 \mathrm{~nm}$, respectively. The different sizes of pigment particles suggest that pigment aggregation happens in pigment dyeing spontaneously [18].

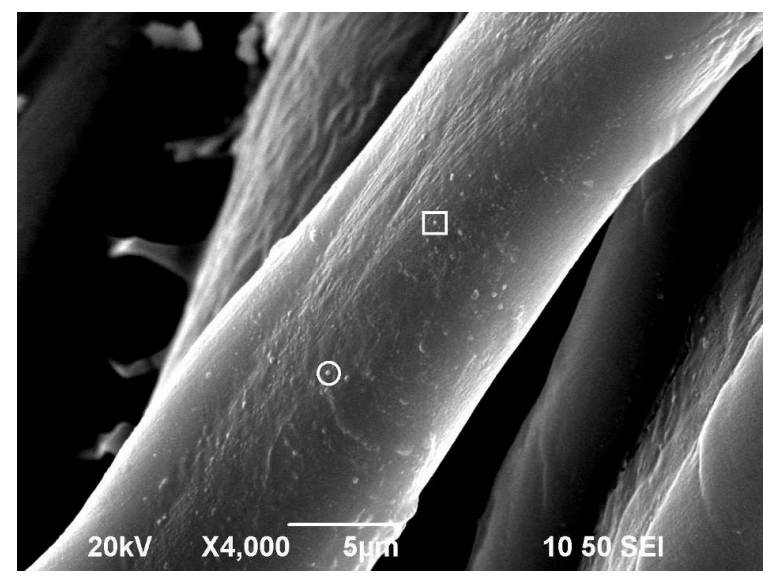

Figure 3. SEM image of plasma treated cotton fiber after pigment dyeing (with pigment agglomeration) (condition: (discharge power $=170 \mathrm{~W}$; oxygen flow rate $=0.4 \mathrm{~L} / \mathrm{min}$; jet moving speed $=5 \mathrm{~mm} / \mathrm{s}$ and jet to substrate distance $=3 \mathrm{~mm}$ ).

\subsection{Crocking Fastness}

The ratings of crocking fastness of pigment dyed samples are presented in Table 4 . The pigment dyed samples have good crocking fastness in dry condition but poorer fastness to wet rubbing. Pigment particles may have strong attractive force only to each other in their solid crystal lattice structure. The attachment of pigment on fibers depends mainly on the mechanical adhesion force between the binder and the fiber surface. Binder's dissolution into water results in serious pigment detachment [2,3]. Moreover, the contact area between specimen and white cloth is larger when water penetrates among fibers. Fabric friction increases when fabric moisture content increases. More pigments are rubbed away by strong mechanical force.

Table 4. Ratings of pigment dyed samples *.

\begin{tabular}{ccccccccccc}
\hline Crocking Fastness & Plasma Untreated & $\mathbf{1}$ & $\mathbf{2}$ & $\mathbf{3}$ & $\mathbf{4}$ & $\mathbf{5}$ & $\mathbf{6}$ & $\mathbf{7}$ & $\mathbf{8}$ & $\mathbf{9}$ \\
\hline Dry & 5 & 5 & 5 & 5 & 5 & 5 & 5 & 5 & 5 & 5 \\
Wet & 3 & $3-4$ & 4 & 4 & $3-4$ & 4 & 4 & 4 & 4 & $3-4$ \\
\hline
\end{tabular}

* Grey scale: 5 and 1 represent the best and the worst color fastness rating, respectively.

Obviously, plasma treatment significant improves wet crocking fastness and the improvement is proportionate to discharge power and oxygen flow rate and inversely proportionate to jet moving speed. Under suitable plasma treatment conditions, the fiber surface is extensively modified by active plasma species. The linkage of polymer-pigment-binder is strengthened by the cracks and grooves physically as more binder molecules can attach on fiber surface when the total surface area is large. As shown in Figure 4, small sized pigment particles $(184.6 \mathrm{~nm})$ are fitted into the slits blocked by the binder layer to resist rubbing. However, the crocking $\mathrm{K} / \mathrm{S}$ values of $150 \mathrm{~W}$ and $170 \mathrm{~W}$ power plasma treated fabrics are similar; further increase of fiber roughness cannot reinforce the mechanical layer and shield pigment particles in a better way in pigment dyeing. Under high power level, high 
oxygen content (high oxygen flow rate) facilitates stronger plasma effect by producing more active species. Since the oxygen content and fiber roughness changes caused by difference in oxygen flow rates are small, reduction of pigment detachment is improved only slightly. Besides discharge power, jet moving speed also exhibits strong influence on crocking fastness. Long treatment times diminish pigment detachment because of the increasing number and width of cleavages.

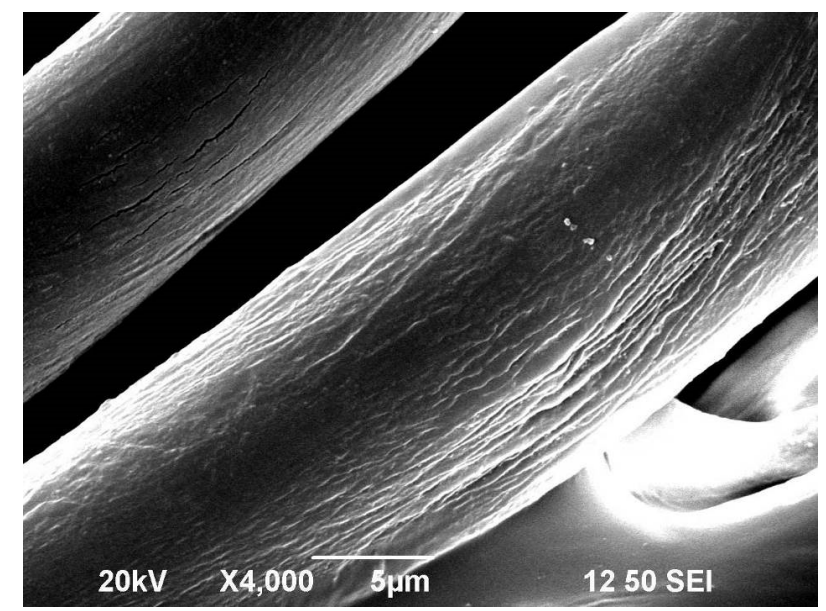

Figure 4. SEM image of plasma treated cotton after pigment dyeing (condition: (discharge power $=170 \mathrm{~W}$; oxygen flow rate $=0.4 \mathrm{~L} / \mathrm{min}$; jet moving speed $=5 \mathrm{~mm} / \mathrm{s}$ and jet to substrate distance $=3 \mathrm{~mm})$.

In dark shade pigment dyeing, plasma treatment significantly improves crocking fastness, however, discharge power, oxygen flow rate, and jet moving speed causes different influences on the final dyeing results.

\subsection{Dry Cleaning Fastness}

The pigment provided by the dye manufacturer has good dry cleaning fastness, $4-5$ (in both color change and staining; Grey scale 5 and 1 represent the best and the worst color fastness rating, respectively), is given in dark shade pigment dyeing. The effective diameter of pigment used in this study is $184.6 \mathrm{~nm}$ which is regarded as micro-sized pigment. Although micro-sized pigments cannot diffuse into the fiber's center, they can be fitted into the cracks and shielded by the binder layer well [19]. As a result, dry-cleaning fastness of plasma treated and untreated samples is very good; only a few loose blue pigment particles are removed in the dry cleaning process. The grey scales of staining on multi-fiber stripes are the same for control and plasma treated fabric samples. Thus, the plasma process would not produce adverse effects on the dry cleaning fastness of dark shade samples.

\section{Conclusions}

Atmospheric pressure plasma treatment using oxygen gas shows a positive influence on color yield of dark shade pigment dyeing in this study. The plasma treatment can increase the color yield and improve the levelness of the pigment dyed cotton fabric. A significant improvement in wet crocking fastness of pigment dyed cotton samples was obtained after treating by plasma. The improvement was due to the change of fiber surface roughness and functionality. Suitable control of discharge power $(150 \mathrm{~W})$, oxygen flow rate $(0.4 \mathrm{~L} / \mathrm{min})$, and jet moving speed $(5 \mathrm{~mm} / \mathrm{s})$ in plasma treatment causes good modification of crocking fastness of cotton fabric. The dry cleaning fastness of pigment dyed fabric samples was good.

Acknowledgments: The authors wish to express their gratitude towards The Hong Kong Polytechnic University for providing the financial support for this work. 
Author Contributions: Chi-Wai Kan proposed the research topic; Chi-Wai Kan and Wai-Shan Man conceived and designed the experiments; Wai-Shan Man performed the experiments; Chi-Wai Kan and Wai-Shan Man analyzed the data; Chi-Wai Kan contributed reagents/materials/analysis tools; Wai-Shan Man wrote the paper and Chi-Wai Kan provided technical advices on the paper before submission.

Conflicts of Interest: The authors declare no conflict of interest.

\section{References}

1. Aspland, J.R. Reactive dyes and their application. Text. Chem. Color. 1992, 5, 31-36.

2. Kan, C.W.; Man, W.S.; Ng, S.P. A study of pigment application on atmospheric pressure plasma treated cotton fabric. Fibers Polym. 2014, 15, 2313-2318. [CrossRef]

3. Man, W.S.; Kan, C.W.; Ng, S.P. The use of atmospheric pressure plasma treatment on enhancing the pigment application to cotton fabric. Vacuum 2014, 99, 7-11. [CrossRef]

4. Bhat, N.V.; Benjamin, Y.N. Surface resistivity behavior of plasma treated and plasma grafted cotton and polyester fabrics. Text. Res. J. 1999, 69, 38-42. [CrossRef]

5. Wang, C.X.; Qiu, Y.P. Two sided modification of wool fabrics by atmospheric pressure plasma jet: Influence of processing parameters on plasma penetration. Surf. Coat. Technol. 2007, 201, 6273-6277. [CrossRef]

6. Morent, R.; De Geyter, N.; Verschuren, J.; De Clerck, K.; Kiekens, P.; Leys, C. Non-thermal plasma treatment of textiles. Surf. Coat. Technol. 2008, 202, 3427-3449. [CrossRef]

7. Černákorá, L'.; Kováčik, D.; Zahoránová, A.; Černák, M.; Mazúr, M. Surface modification of polypropylene non-woven fabrics by atmospheric-pressure plasma activation followed by acrylic acid grafting. Plasma Chem. Plasma Process. 2005, 25, 427-437.

8. Cai, Z.S.; Qiu, Y.P. The mechanism of air/oxygen/helium atmospheric plasma action on PVA. J. Appl. Polym. Sci. 2006, 99, 2233-2237. [CrossRef]

9. Kan, C.W.; Yuen, C.W.M.; Tsoi, W.Y. Using atmospheric pressure plasma for enhancing the deposition of printing paste on cotton fabric for digital ink-jet printing. Cellulose 2011, 18, 827-839. [CrossRef]

10. Sun, Y.; Liang, Q.; Chi, H.; Zhang, Y.; Shi, Y.; Fang, D.; Li, F. The application of gas plasma technologies in surface modification of aramid fiber. Fibers Polym. 2014, 15, 1-7. [CrossRef]

11. Haji, A.; Qavamnia, S.S. Response surface methodology optimized dyeing of wool with cumin seeds extract improved with plasma treatment. Fibers Polym. 2015, 16, 46-53. [CrossRef]

12. Teli, M.D.; Samanta, K.K.; Pandit, P.; Basak, S.; Chattopadhyay, S.K. Low-temperature dyeing of silk fabric using atmospheric pressure helium/nitrogen plasma. Fibers Polym. 2015, 16, 2375-2383. [CrossRef]

13. Chong, C.L.; Li, S.Q.; Yeung, K.W. An objective method for the assessment of levelness of dyed materials. J. Soc. Dyers Color. 1992, 108, 528-530. [CrossRef]

14. American Association of Textile Chemists and Colorists. Technical Manual of the American Association of Textile Chemists and Colorists; AATCC: Research Triangle Park, NC, USA, 2017; pp. 21-23.

15. American Association of Textile Chemists and Colorists. Technical Manual of the American Association of Textile Chemists and Colorists; AATCC: Research Triangle Park, NC, USA, 2017; pp. 236-238.

16. Kwong, C.H.; Ng, S.P.; Kan, C.W.; Molina, R. Inducing hydrophobic surface on polyurethane synthetic leather by atmospheric pressure plasma. Fibers Polym. 2014, 15, 1596-1600. [CrossRef]

17. Mukhopadhyay, S.M.; Joshi, P.; Datta, S. Plasma assisted surface coating of porous solids. Appl. Surf. Sci. 2002, 201, 219-226. [CrossRef]

18. Chen, Y.; Wang, Z.; Song, F.; Xu, Y. The effect of different plasma treatments on the pigment dyeing of cotton fabrics. Adv. Mater. Res. 2012, 441, 96-101. [CrossRef]

19. Hao, L.Y.; Wang, R.; Liu, J.Q.; Liu, R.Z. Ultrasound-assisted adsorption of anionic nanoscale pigment on cationised cotton fabrics. Carbohydr. Polym. 2012, 90, 1420-1427. [CrossRef] [PubMed]

(C) 2017 by the authors. Licensee MDPI, Basel, Switzerland. This article is an open access article distributed under the terms and conditions of the Creative Commons Attribution (CC BY) license (http:/ / creativecommons.org/licenses/by/4.0/). 